\title{
DEUTERIUM IN THE SOLAR SYSTEM
}

\author{
T. OWEN \\ Institute for Astronomy \\ 2680 Woodlawn Drive \\ Honolulu, HI 96822 \\ USA
}

\begin{abstract}
Values of $\mathrm{D} / \mathrm{H}$ measured in the methane on the giant planets and Titan indicate the presence of two distinct reservoirs of deuterium in the outer solar system. The dominant reservoir is in hydrogen gas, the second, multi-component reservoir is found in the hydrogen that is bound in condensed compounds. Both reservoirs appear to have originated in the interstellar medium. In contrast, the values of $\mathrm{D} / \mathrm{H}$ in water vapor on Mars and Venus (especially) exhibit a large enrichment from the "condensed matter" starting value. Interpretation of this enrichment may illuminate the history of water on these two planets.
\end{abstract}

\section{Introduction}

During the past fifteen years, Drs. Catherine de Bergh, Barry Lutz, Jean Pierre Maillard and I have been engaged in a systematic study of the ratio of $\mathrm{D} / \mathrm{H}$ in planetary and satellite atmospheres. We decided from the outset to determine this isotopic ratio in the outer solar system by studying $\mathrm{CH}_{3} \mathrm{D}$ and $\mathrm{CH}_{4}$, rather than using $\mathrm{HD}$ and $\mathrm{H}_{2}$. This decision was based on our perception that it is extremely difficult to measure equivalent widths of the very weak lines of $\mathrm{HD}$ and $\mathrm{H}_{2}$ in the presence of ubiquitous weak lines of $\mathrm{CH}_{4}$ in the spectra of all of the giant planets and Titan. Smith et al. (1989) have recently described this difficulty in detail.

To study deuterium on the inner planets, we elected to use $\mathrm{HDO}$ and $\mathrm{H}_{2} \mathrm{O}$, as these are the most abundant carriers of hydrogen in the atmospheres of Mars and Venus. We considered using DCl on Venus, but had to abandon this idea because of strong interference from absorptions of $\mathrm{CO}_{2}$ and $\mathrm{CO}$.

\section{THE OUTER SOLAR SYSTEM}

The first step was to make the necessary quantity of $\mathrm{CH}_{3} \mathrm{D}$ and examine the spectrum of this molecule to identify and analyze absorption bands that could be used for our purpose (Danehy et al. 1977, Lutz et al. 1978, 1983). This exercise led to the conclusion that the second overtone of the vibrational frequency $v_{2}$ at $6425 \mathrm{~cm}^{-1}$ was ideal, in that it falls in the $1.6 \mu \mathrm{m}$ window of the $\mathrm{CH}_{4}$ spectrum. Having made this identification, it was possible to find suggestive evidence for the presence of this band in existing spectra of Titan and Uranus that had been recorded by Fink and Larson (1979) at a resolution of $3.6 \mathrm{~cm}^{-1}$ (Lutz et al. 1981).

To progress, we had to obtain our own spectra at a higher resolution of $1.2 \mathrm{~cm}^{-1}$, first of Uranus (de Bergh et al. 1986) and Titan (de Bergh et al. 1988). To these we finally added Neptune at a resolution of $4.0 \mathrm{~cm}^{-1}$ (de Bergh et al. 1990). Along the way, we had studied Satum as a control, since a value of $\mathrm{D} / \mathrm{H}=2.2+1.8 /-1.7 \times 10^{-5}$ had been deduced for this planet by Courtin et al. (1984). These authors used Voyager observations of the $8.6 \mu \mathrm{m}$ methane band complex in 
emission to derive this result, a technique which had been employed successfully on Jupiter by Kunde et al. (1982). We obtained $\mathrm{D} / \mathrm{H}=1.7=+1.7 /-0.8 \times 10^{-5}$ for Saturn, suggesting that our method did not introduce any large systematic errors. We could not use our approach on Jupiter because absorption bands of ammonia block the window at $1.6 \mu \mathrm{m}$ where our $\mathrm{CH}_{3} \mathrm{D}$ band occurs.

The results of this investigation are summarized in Figure 1, which includes values of $\mathrm{D} / \mathrm{H}$ determined in $\mathrm{CH}_{4}$ on Jupiter (discussed by Gautier and Owen 1989), and an updated value of $\mathrm{D} / \mathrm{H}=1.7 \pm 1.1 \times 10^{-5}$ for Saturn (Noll and Larson 1991). The value of $\mathrm{D} / \mathrm{H}=1 \pm 0.5 \times 10^{-5}$ for the Interstellar Medium is based on the discussion by $R$. Ferlet at this conference. The protosolar value of $3.0 \pm 1.0 \times 10^{-5}$ is derived from solar ${ }^{4} \mathrm{He} / \mathrm{H}=0.097$ and meteoritic ${ }^{3} \mathrm{He} /{ }^{4} \mathrm{He}$ $=1.4 \pm 0.2 \times 10^{-4}$ given by Anders and Grevesse (1989) and solar wind ${ }^{3} \mathrm{He} /{ }^{4} \mathrm{He}=4.4 \pm 0.5 \times$ $10^{-4}$ from Bochsler et al. (1990).

We conclude from inspection of Figure 1 that there are two principal reservoirs of deuterium in the outer solar system (Owen et al. 1986). The largest reservoir consists of the hydrogen gas that originally dominated the solar nebula and is now the main constituent of Jupiter and Satum. This should be the protosolar value of $\mathrm{D} / \mathrm{H}$ and indeed, the Jupiter and Saturn average of $\mathrm{D} / \mathrm{H}=2.0 \pm$ $1.0 \times 10^{-5}$ overlaps the value of $\mathrm{D} / \mathrm{H}=3.0 \pm 1.010^{-5}$ derived from meteorites and the solar wind. The second reservoir must actually consist of several different species of condensed hydrogencontaining compounds, presumably dominated by $\mathrm{H}_{2} \mathrm{O}$. Thus the value of $\mathrm{D} / \mathrm{H}$ that we measure in gas from this reservoir must be an average of several different values set by ion-molecule and grain-catalyzed reactions in the interstellar medium. We find evidence for this reservoir in the methane on Titan, Uranus and Neptune. A high value of $\mathrm{D} / \mathrm{H}$ resulting from the mixing of material from the icy cores of these planets was predicted long ago by Hubbard and MacFarlane (1980). These predictions are illustrated in Figure 1 by an " $x "$.

\section{THE INNER SOLAR SYSTEM}

The condensed matter reservoir is also manifested in the high value of $\mathrm{D} / \mathrm{H}$ seen in $\mathrm{H}_{2} \mathrm{O}$ in meteoritic hydrated silicates (Yang and Epstein 1983), and in Halley's Comet (Eberhardt et al., 1987) as illustrated in Figure 1. Still higher values are found in some of the organic compounds identified in carbonaceous chondrites (Yang and Epstein 1983, Epstein et al. 1987) and in interplanetary dust particles (Zinner et al. 1983) (see Figure 1). Given the meteoritic and cometary bombardment of the early inner planets, we may reasonably expect that the condensed matter reservoir fumished the starting value of $\mathrm{D} / \mathrm{H}$ on all of these bodies. On Earth, we find that the present value of $\mathrm{D} / \mathrm{H}$ in the oceans is overlapped by the various determinations of this ratio in objects representing the condensed matter reservoir (Figure 1).

Some slight enrichment of $\mathrm{D} / \mathrm{H}$ on Earth must have occurred as a result of the dissociation of $\mathrm{H}_{2} \mathrm{O}$ and resulting hydrogen escape (Yung et al. 1989). Studying $\mathrm{H}_{2} \mathrm{O}$ and $\mathrm{HDO}$ on Mars and Venus, we found values of $\mathrm{D} / \mathrm{H}$ that are respectively $6 \pm 3$ and $120 \pm 40$ times the telluric values (Owen et al. 1988, de Bergh et al. 1991). The Martian value has since been confirmed by Bjoraker et al. (1989) who obtained an enrichment of $5.2 \pm 0.2$ times telluric, while the 100 -fold enrichment of D/H on Venus was originally discovered by McElroy et al. (1982) and Donahue et al. (1982) with mass spectrometers on the Pioneer Venus Spacecraft.

The interpretation of these enrichments is complicated by the variety of processes that are involved: the addition of water by cometary impact, chemical reactions with surface materials, changes in the luminosity of the sun, etc. The original interpretation of the Venus enrichment by 


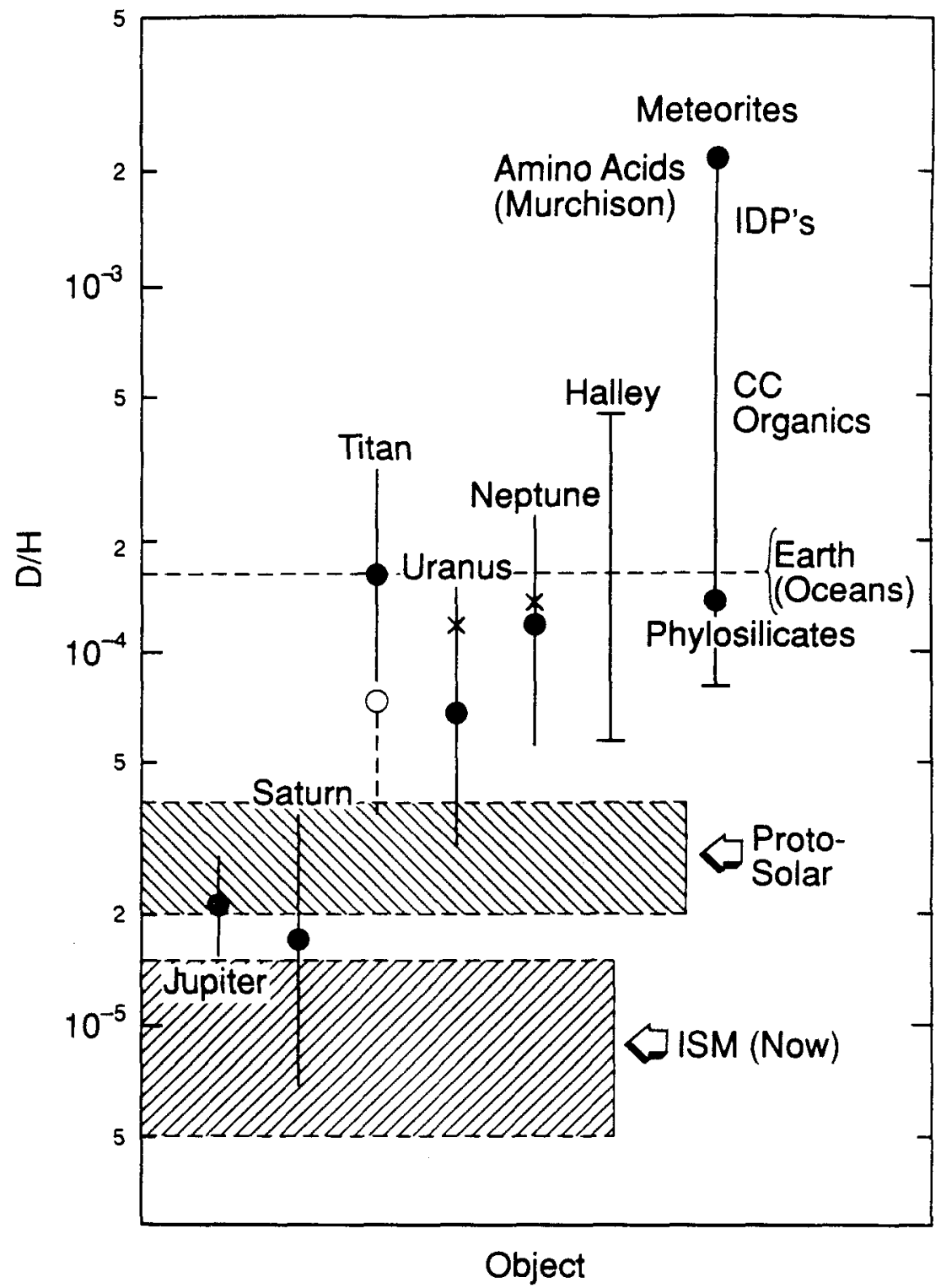

Figure 1: Values of $\mathrm{D} / \mathrm{H}$ as measured in various bodies in the solar system compared with the present interstellar value and the protosolar value deduced from meteorites and the solar wind. The open circle shown for Titan represents a correction for enrichment of $\mathrm{D} / \mathrm{H}$ through atmospheric escape. 
Donahue et al. (1982) in terms of a runaway greenhouse that depleted a starting inventory of $\geq$ $0.3 \%$ of the Earth's oceans still seems the most reasonable scenario (de Bergh et al. 1991 - see Carlson et al. 1991 for a new value of the global $\mathrm{H}_{2} \mathrm{O}$ abundance that reinforces this conclusion). The Martian atmosphere is so thin that a single cometary impact has the potential of re-setting the hydrogen isotopes (Owen 1992). Thus it is difficult to know how significant the present D/H value is for the long-term history of the planet.

\section{CONCLUSIONS}

The results for the outer planets suggest that the hydrogen gas in the atmospheres of Jupiter and Saturn can be used to obtain an accurate measurements of the solar nebula value of $\mathrm{D} / \mathrm{H}$. In effect, this will be a measurement of $\mathrm{D} / \mathrm{H}$ in an interstellar cloud as it existed 4.5 billion years ago. Compared with measurements of the present value of $\mathrm{D} / \mathrm{H}$ in the ISM, this value can be used in principle to constrain models for big-bang nucleosynthesis and galactic evolution, particularly when combined with an accurate value of ${ }^{3} \mathrm{He} /{ }^{4} \mathrm{He}$. Both ratios can be determined by the mass spectrometer on the Galileo Probe, now set for delivery into the Jovian atmosphere in December 1995 (Niemann et al. 1992).

The relatively high value of $\mathrm{D} / \mathrm{H}$ measured in Titan's methane excludes models for the origin of this satellite's atmosphere that involve reduction of interstellar $\mathrm{CO}$ to $\mathrm{CH}_{4}$ in the Saturn subnebula (Owen and Gautier 1989). It seems more likely at this point that the methane comes from the decomposition of organic compounds (grains) during the accretion of the satellite.

Finally, there is much more to be done before the interpretation of the high values of $\mathrm{D} / \mathrm{H}$ on Venus and Mars will be completed. The outstanding problem on Venus is to obtain a reliable value for the global atmospheric water abundance. This is possible from Earth-based observations, and may even be achieved this year. On Mars, we need additional information on oxygen isotopic ratios in the atmosphere and in the rocks as well as in situ measurements of D/H in sub-surface and polar ice. Only then will we be able to unravel the time-history of the atmosphere with any confidence.

\section{References}

Anders, E., and Grevesse, N. 1989, Geochim. et Cosmochim. Acta, 53, 197-214.

Bjoraker, G. L., Mumma, M. J., and Larson, H. P. 1989, Bull. Amer. Astron. Soc., 21, 990 (abstract).

Bochsler, P., Geiss, J., and Maeder, A. 1990, Solar Physics 128, 203-215.

Carlson, R. W., et al. 1991, (Science in press).

Courtin, R., Gautier, D., Marten, A., and Bèzard, B. 1984., Astrophys. J., 287, 899-916.

de Bergh, C., Bèzard, B., Owen, T., Crisp, D., Maillard, J. P., and Lutz, B. L. 1991, Science, 251. 547-579.

de Bergh, C., Lutz, B. L., Owen, T., Brault, J., and Chauville, J. 1986, Astrophys. J., 311, 501510.

de Bergh, C., Lutz, B. L., Owen, T., and Chauville, J. 1988, Astrophys. J., 329, 951-955.

de Bergh, C., Lutz, B. L., Owen, T., and Maillard, J. P. 1990, Astrophys. J., 355, 661-666. 
Danehy, R. G., Lutz, B. L., Owen, T., Scattergood, T. W. and Goetz, W. 1977, Astrophys. J. Lett., 273, 397-409.

Donahue, T. M., Hoffman, J. H., Hodges, R. R. Jr., and Watson, A. J. 1982, Science 216, 630633.

Eberhardt, P., Dolder, U., Schulte, W., Krankowsky, D., Låmmerzahl, P., Hoffmann, J. H., Hodges, R. R., Berthelier, J. J., and Illiano, J. M. 1987, Astron. Astrophys., 187, 435437.

Epstein, S., Krishnamurthy, R. V., Cronin, J. R., Pizzarello, S., and Yuen, G. U. 1987, Nature, 326, 477-479.

Fink, U., and Larson, H. P. 1979. Astrophys. J., 233, 1021-1040.

Gautier, D., and Owen, T. 1989, in Origin and Evolution of Planetary and Satellite Atmospheres, ed. S. K. Atreya, J. B. Pollack, and M. S. Matthews (Tucson: U. of Arizona press) 487512.

Hubbard, W. B. and MacFarlane, J. J. 1980, Icarus, 44, 676-682.

Kunde, V. G., Hanel, R. A., Maguire, W. C., Gautier, D., Baluteau, J. P., Marten, A., Chedin, A., Husson, N., and Scott, N. 1982, Astrophys. J., 263, 443-467.

Lutz, B. L., de Bergh, C. and Maillard, J. P. 1983, Astrophys. J., 273. 397-409.

Lutz, B. L., Danehy, R. G., and Ramsay, D. A. 1978, J. Molec. Spectrosc. 72, 128.

McElroy, M. B., Prather, M. J., and Rodriquez, J. 1982, Science, 215, 1614-1615.

Niemann, H. B., Harpold, D. N., Atreya, S. K., Carignan, G. R., Hunten, D. M., and Owen, T. C. 1992, Space Sci. Rev. (in press).

Noll, K. S., and Larson, H. P. 1991, Icarus 89, 168-189.

Owen, T. 1992, in MARS, ed. H. Kieffer, B. Jakosky, and M. Matthews (Tucson: U. of Arizona Press) (in press).

Owen, T. and Gautier, D. 1989, Adv. Space Res. 9, (2)73-(2)78.

Owen, T., Lutz, B. L., and de Bergh, C. 1986, Nature 320, 244-246.

Owen, T., Maillard, J. P., de Bergh, C., and Lutz, B. L. 1988, Science, 240, 1767-1770.

Smith, W. H., Schempp, W. V., Simon, J., and Baines, K. H. 1989, Astrophys. J. 336, 962.

Yang, J. and Epstein, S. 1983, Geochim. et Cosmochim. Acta, 47, 2199-2216.

Yung, Y. L., Wen, J.-S., Moses, J. I., Landry, B. M., Allen, M. and Hsu, K.-J. 1989, J. Geophys. Res., 94, 14971-14989.

Zinner, E., McKeegan, K. D., and Walker, R. M. 1983, Nature 305, 119-121. 